\title{
Designing An ApPlication To Optimize The Calibration Of OUTPUT DeVices In Digital Print EnVIRonMent
}

\author{
Milan Navrátil \& Jaromír Polášek
}
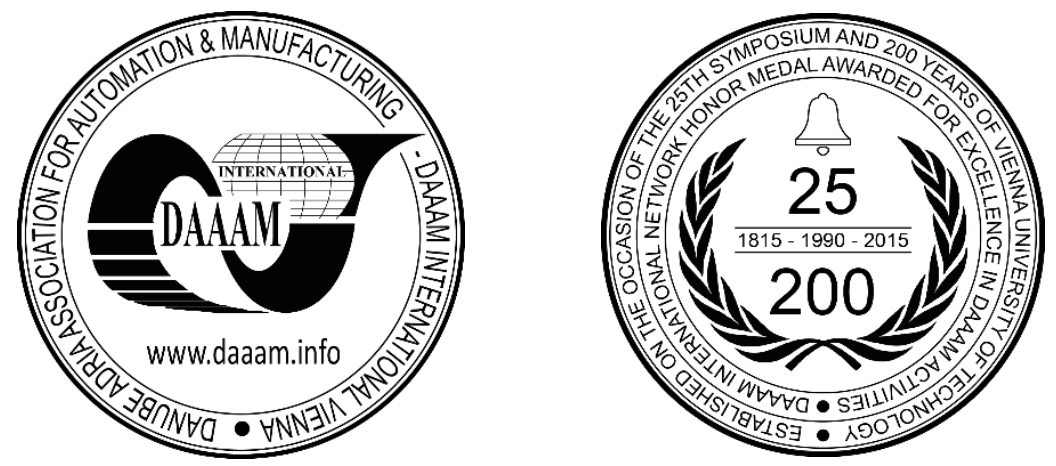

This Publication has to be referred as: Navratil, M[ilan] \& Polasek, J[aromir] (2017). Designing An Application To Optimize The Calibration Of Output Devices In A Digital Print Environment, Proceedings of the 28th DAAAM International Symposium, pp.0244-0252, B. Katalinic (Ed.), Published by DAAAM International, ISBN 978-3-90273411-2, ISSN 1726-9679, Vienna, Austria

DOI: $10.2507 / 28$ th.daaam.proceedings.033

\begin{abstract}
Aim of this paper is analysis of measured data from spectrophotometric probe and design of methods applicable in printer operation for the optimization of linearization curves. The paper also deals with application design in Microsoft .NET platform to optimize the calibration of output devices in a digital print environment. The proposed application is based on both, the initial linearization requirements, i.e. before creating an ICC profile, as well as the possibilities of relinearizing the printer to extend the life of the profiles. The statistical analysis, description of measured data, calculation of colour differences and possibilities that linearization for colour management offers are also described. The proposed application helps to eliminate the serious technological problems in the creation of output-ICC profiles just for the needs of digital printing machines and other output devices with CMYK output and with regard to the technology of sublimation of these colours on textile materials.
\end{abstract}

Keywords: Spectrophotometer; measurement; linearization; digital printing; .NET; application

\section{Introduction}

Current requirements for the operation of printers and printing plants generally place great emphasis on true colour processing as well as on output quality. Due to the increasing development of computer technology, the possibilities in the area of source data processing as well as the control of the output device as such are increased. Hand-in-hand with the development of new technologies, new challenges and related problems and obstacles are coming.

With the need for colourful fidelity, demands not only for accuracy but also for its own sustainability arise. The accuracy is assured with the well-established output profile. In this case, sustainability is meant to maintain the output profiles and to increase their lifetime. Materials and colours commonly available on the industrial processing market show some degree of variability in terms of their attributes within the range indicated by the manufacturer. Parameters may vary depending on the current production series, sub suppliers, regulatory or market requirements. Operation must be able to react flexibly to these changes and regularly re-calibrate the output profiles or create new ones.

Frequently neglected or mitigated technologies include linearization, which should be an integral part of creating and maintaining the output profile of the device. However, the current Raster Image Processor (RIP) tools and their equipment are only marginally devoted to this area and offer very little or no analysis or back testing. Some of them even encourage user in order to influence the measured values. This can lead to fatal consequences for back testing or re-linearization, as 
the same starting conditions are not maintained. The operator has modified the results, so they cannot agree with the same measurement in another time or by a measurement made by another person. Such a measurement does not reflect the real state of the machine but rather the ideal idea of the operator, based on his experience.

One of the problematic technologies for linearization is sublimation printing, which in the printing industry represents considerable potential in the field of printing of advertising articles and various textiles, both ordinary needs and specific designations. By introducing this technology, which significantly differs from the standard direct digital printing, at least in the way of its application, the tools used for maintaining and calibrating printers, possibly for output profiles, have appeared to be inadequate. Where existing tools have had rather shortcomings, sublimation has begun to show complete inability to make linearization at all. The measured values were noisy. After the introduction of this technology, even after several attempts and attempts to measure as accurately as possible and to observe the conditions set by the probe manufacturer, the linearization, especially the yellow channel, failed to be usable. This has fully demonstrated the need for an analytical tool to help reveal the problem or even to solve it. One of the solutions to this issue can be good analytical tools, which are already commercially available today, but with their educational requirements far exceed the knowledge and capabilities of the average machine operator. By repeating the procedure the operator chooses, the input and output conditions must be maintained. The entire operation must be repeatable, if necessary, with the same results or with differences caused by any changes in the parameters of the packing of the output device or of the print media itself.

Alternatively, the measuring device is a so-called spectrophotometric probe with a higher resolution or a smaller deviation. This solution, in addition to higher acquisition costs, brings further problems in terms of compatibility and widespread use. Top-class devices are designed and delivered with corresponding application-specific software especially for industrial use in production. From these reasons and due to their cost these devices cannot be often used. Operational requirements for these devices are also much higher than the requirements for an industrial print operator. The solution, therefore, should be to process data from a commonly used device that can be used with a wide range of RIPs and Profilers.

The last possible solution to this problem can be a simple analytical tool, capable of graphically displaying and archiving the measured values and the results of several measurements between them, or averaging the data so that the same input and output conditions are maintained for each linearization. This tool, if it retains the concept of simplicity and availability, could be a great help not only in large businesses, but also small in printers with limited portfolio that are interested in quality and sustainable production.

\section{Digital print}

The term digital printing includes a number of printing techniques. Its main feature is direct printing from digital data. This eliminates the preparation outside of the printing machine. In the case of digital printing, the machine only has the required print media. All that is needed for printing either arises directly in the machine or is a part of it. Digital printing works on a similar principle as desktop printers. The devices have their own RIP or a third party RIP that sorts print jobs and rasters print data. From the point of view of the principle, the most commonly used technologies are electrographic and jet or inkjet printing (so-called ink-jet). Powder or liquid toners or inks are used as the color. The advantage of this technology is minimal pre-printing and final output. Compared to other technologies, for example, the drying time is negligible [1], [2].

From a technical point of view, this is a very sensitive process that depends on many factors, such as the type and model of the output device, the type of media to which printing is applied, the ink setting, the resolution, the half toning method, print speed, output quality, preheat and media drying and more. Changing any of these attributes results in a change in appearance but also the color of the output. For this reason, it is necessary to check and record the environment in which the machine has been calibrated and, if any, change its recalibration. For example, simply changing the paperweight can completely change its water absorption and affect the overall output [1].

\subsection{Calibration of digital print machines}

Successful color reproduction across different materials and media requires calibration and color characterization of input and output devices. The calibration process will place the device in an up-to-date, unchanging condition that does not change or change over a certain range during the use of the device within a specified period. Characterization, on the other hand, de- fines the model of the input and output values of the device [3], [4].

Digital printing on textiles, compared to direct printing on a medium, requires much more production time. Printing, for example, on paper is relatively easy, but printing on textiles often requires further steps of the overall process such as sublimation or subsequent washing of textiles to remove remnants of non-dyed paint. The calibration of the output device for textile printing in one-step would be an effective way. However, this method is not suitable or feasible, since the overall calibration quality will be low. The two-step process is a good compromise between the quality of calibration and usability in real traffic on the other hand. The first step of the calibration is linearization and the second output of the output profile itself [1], [3], [5].

\subsection{Linearization}

Calibration of one color output channel is a basic and often neglected process of initial setting of the output device. The disadvantage of half-toning techniques used by modern printers is non-linear output. With regard to the most 
widespread techniques such as "dithering", "error diffusion" or "stochastic screening", the issue becomes even more complex. The use of modern headsets with variable droplet size and Light-Ink usage still contributes to the rise in nonlinear output. The process consists in printing a calibration card with precisely defined fields, with the increment of each individual color and its subsequent measurement. The result is then displayed and can be manually adjusted if necessary [1], [6], [7].

The main aim of linearization is therefore the color characteristics of the output from the printer and the conversion of color information from the independent PCS color space into CMYK comprehensible to the printer. Since CIELAB and CMYK are not linearly dependent, there is a reason to create a linear approximation. Thus, linearization can be understood as the mathematical model of the output device as follows: [6]

$$
(\mathrm{C}, \mathrm{M}, \mathrm{Y}, \mathrm{K})=f\left(L^{*}, a^{*}, b^{*}\right)
$$

This is one way to minimize the error between Original CMYK values and model values. Linearization of CMYK values into the CIELAB space will facilitate the colour characterization of the output device in many cases. Another reason for linearization is the often-used 3D Output Profiling Method called 3D LUT. Output profiles contain a 3D table defining a colour transformation from CIELAB to CMYK. Generally, it consists of a uniform 3D grid in CIELAB colour space. For each printed pixel, the colour transformation is calculated by linear interpolation between the values in the grid using interpolation patterns. This scheme can be a tri-linear or tetrahedral interpolation. Because conversion from CIELAB to CMYK is strongly nonlinear, this interpolation can lead to minor errors, especially if 3D LUT is defined on a very coarse grid. Linearizing CMYK values into CIELAB space using 1D LUT will convert more linearly using 3D LUT [6].

An important feature of linearization of printing machines is the resulting non-linearity. We are talking about getting non-completely linear output. Because the measured magnitude of most RIP and linearization tools is luminescent component $\mathrm{L}^{*}$, measured value 50 does not mean $50 \%$ coverage [1], [8].

The CIELAB color space is a $3 \mathrm{D}$ grid that has the $\mathrm{L}^{*}, \mathrm{a}^{*}$ and $\mathrm{b}^{*}$ axes. For measurements for linearization purposes, the following equation is offered:

$$
D=\sqrt{\left(L^{\prime}\right)^{2}+\left(a^{\prime}\right)^{2}+\left(b^{\prime}\right)^{2}}
$$

Where $L^{\prime}=\max \left(L^{*}\right)-L^{*}, a^{\prime}=\max \left(a^{*}\right)-a^{*}, b^{\prime}=\max \left(b^{*}\right)-b^{*}$. The problem of this solution lies in the higher sensitivity of the human eye to the luminescent component $\left(\mathrm{L}^{*}\right)$ than to the chromatic $\left(\mathrm{a}^{*}\right.$ and $\left.\mathrm{b}^{*}\right)$. The sum of all three components with the same weight will not result in a linear result. Another option is to use only the $\mathrm{L}^{*}$ folder. For reasons of mesh sensitivity and positive values only, it seems to be the best solution. This solution will work with CMK channels, but for the Y channel, the yellow color is the dynamic range of the $\mathrm{L}^{*}$ axis significantly smaller. Thus, the result on the $\mathrm{L}^{*}$ axis will not yield satisfactory results. On the other hand, the $\mathrm{b}^{*}$ axis is the largest in the yellow color.

\subsection{Re-Linearization}

Re-linearization is intended to recreate the output device model in order to correct errors resulting from its use. As time passes, the attributes of the output device change, reducing the quality of the output and its colour fidelity, i.e., match to the source. One option is to create a new device linearization. Another factor may be variations in the batch of fillings or print media. Generally, manufacturers recommend that they be linearized with each change of charge or a severe change in operating conditions. Re-linearization can bring the device with the output profile used so far to the state in which it was created when creating the first linearization and output profile without having to re-create the profile. Compared with profile formation, the linearization process is much less time consuming. Another possibility is a completely new linearization of the device. This operation is more time-consuming, but still incomparably less time consuming than creating a new profile. It is necessary to take into account that re-linearization or new basic linearization will remove only minor deviations. It cannot be expected that an error of magnitude $\Delta \mathrm{E}$ of 3.2 will create an error $\Delta \mathrm{E}=1$ [9], [10].

\section{Measurement and data preparation}

Textile materials with non-standard behaviour was selected. Additionally, the number of samples of each material was determined, and the measured data was subjected to statistical methods in StatSoft Statistica software. Measurements was accomplished using the third-party Roland WersaWorks Advanced RIP software supplied with the printer. The reasons for using this RIP are the possibility of direct communication with the printer and the export of data to $c s v$ format, which is easy to import into Statistica for subsequent data analysis.

\subsection{Sample preparation}

Print run on the Roland Versa Art RS-640 in default mode at 540 x 360 dpi, with three passes. Air conditioning in the printer room maintains a standard temperature of $18^{\circ} \mathrm{C}$ and a relative humidity in the range of $35-40 \%$. For our measurements, the temperature was raised to the desired $23{ }^{\circ} \mathrm{C}$. Head feed rate was $964 \mathrm{~cm} . \mathrm{min}^{-1}$; feed rate was $30 \mathrm{~cm} \cdot \mathrm{min}^{-1}$ (rates are specified by the machine manufacturer). Drops dropped onto transfer paper heated to $45^{\circ} \mathrm{C}$; the 
paper finished the drying at the same temperature. The machine was not equipped with airflow fans. Transfer to final materials was set after 24-hour technological break. Transfer was carried out on the machine from GMC Company. Next day, the machine was set to a temperature of $195^{\circ} \mathrm{C}$ and, after reaching this temperature; the prints were transferred to eight textile materials (see Table 1).

\begin{tabular}{|c|c|c|}
\hline Mark / Stock weight $\mathbf{( g / \mathbf { m } ^ { \mathbf { 2 } }}$ & Using & Material, Composition (\%) \\
\hline BLOCKOUT / 270 & both-sided flags & PES 100 \\
\hline COOLMAX / 200 & thermos clothes & PES 100 \\
\hline DOUBLE / 160 & sports dresses, t-shirts & PES 50, COOLMAX 50 \\
\hline LYCRA / 200 & elastic clothes & PES 88, ELASTAN 12 \\
\hline SATIN / 135 & pillows, dresses & PES 100 \\
\hline GARDEX / 172 & tents, awnings 100 \\
\hline UTEX / 69 & inflatable items & PES, NYLON \\
\hline POLYFLAG & flags, banners & PES 100 \\
\hline
\end{tabular}

Table 1. List of measured fabrics and materials

\subsection{Measuring process}

The spectrophotometer X-rite ilpro2 from Gretag MacBeth Company was used for colour measurement of the selected samples. This instrument is supported by many third party applications and from the point of view of the scan quality, is a good choice between quality and price and it is suitable for our purposes. The documentation lists the maximum and average differences between the measurements of twelve BCRA (British Ceramic Research Association) tiles. Differences are reported in $\Delta \mathrm{E} 94 *$ at $23{ }^{\circ} \mathrm{C}$ for D50 lighting and $2^{\circ}$ angle as follows: $\Delta \mathrm{E} 94 *_{\max }=1.0$ and $\Delta \mathrm{E} 94 *_{\text {avg }}=0.4$ Documentation also provides a procedure for short-term repeatability where $\Delta \mathrm{E} 94^{*}=0.1$ on a white surface (not specified) for D50 mode at an angle of $2^{\circ}$, at ten repetitions every 3 seconds [11].

Before starting the measurement process it is necessary to determine the optimal number of measurements. If we proceed from the repeat measurement procedure that shows the smallest difference $\Delta \mathrm{E}$, the best adherent is ten repetitions for each material. It is customary to perform five measurements at the first linearization and only three measurements for control or re-linearization. By selecting ten measurements, we obtain a sufficient data reserve for extreme values. All measurements were made on lanes consisting of 21 samples with size 10 x $10 \mathrm{~mm}$ as shown in Fig. 1.

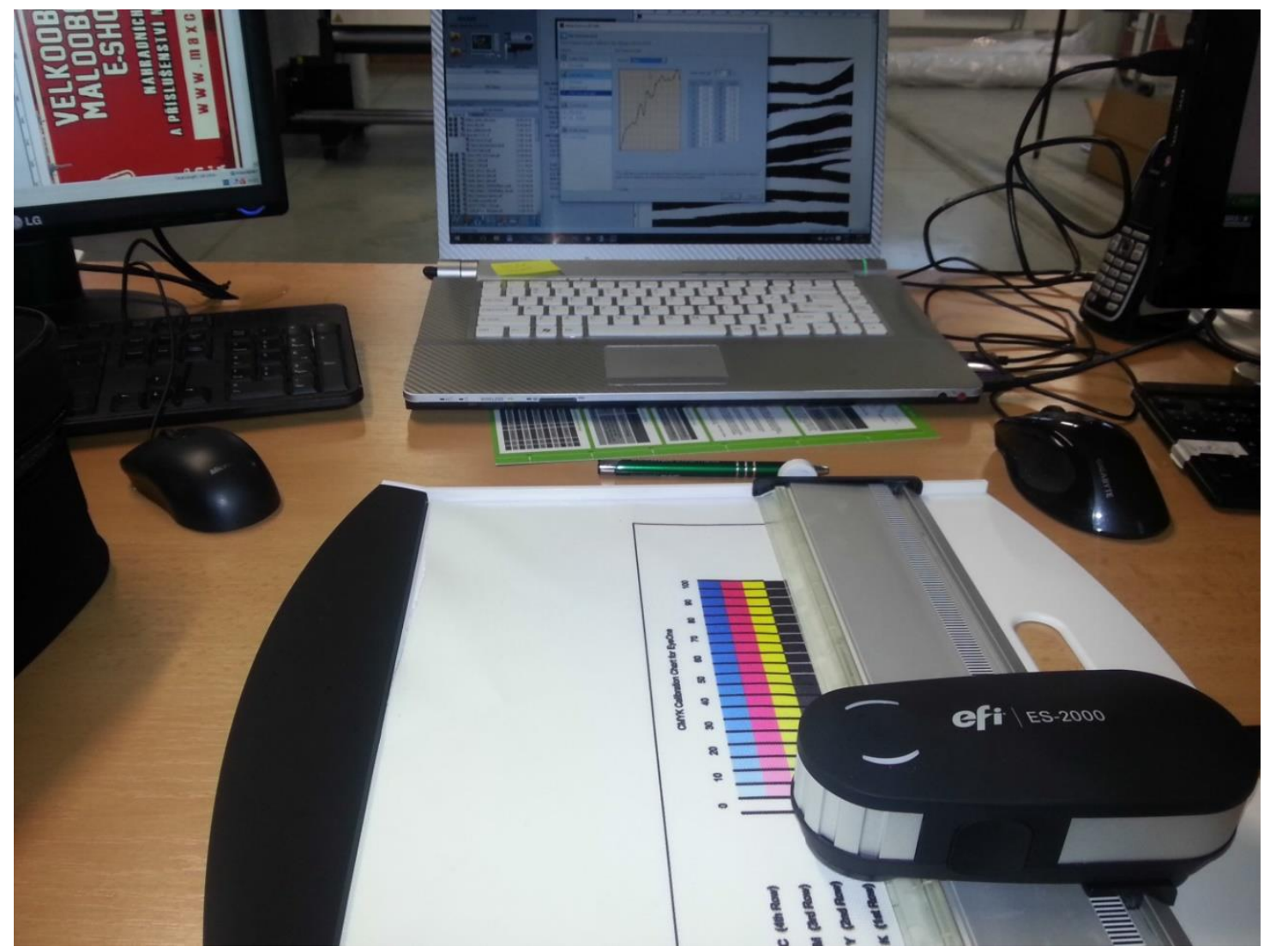

Fig. 1. Measurement of prepared CMYK testing target of a particular sample. 
Each copy of the material was measured with the spectrophotometer and then the data was numbered and divided into folders by names for further analysis and visualization.

\section{Design of user application}

In order to create a usable application, it is necessary to define a list of requirements that will further guide the development of the application. This list serves as a guideline for designing individual components and logic of the entire application. It makes it possible to define clearly not only the framework in which the application will work, but also the means of support that it will require and the way, how it will be developed. Microsoft Visual Studio 2015 development environment was used.

\subsection{Application requirements}

\section{Overview of non-functional requirement}

The application must be capable of being usable in the operation for which it is designed. They have to meet their demands and requirements to fit existing equipment and at the same time maintain a certain degree of versatility. Its demands and requirements cannot exceed the standard equipment of a regular printer and its software tools. Most RIPs are running on Windows. On Mac platforms, it is more likely to prepare graphics as such than directly to print.

The first non-functional requirement, as mentioned in the previous paragraph, will be the MS Windows operating system in the currently still supported version 7. Advantage of maintaining the same platform as that of the RIPs is concurrency on a single PC. Therefore, you can switch between applications, you will not have to use another PC or run another operating system. The ".NET Framework" is available as an application runtime environment. This extensive software platform is designed to develop many kinds of different applications. It allows programming in several languages (e.g. C \#, F \#, J \# VB). All these languages are translated into runtime itself (CLR) at start up. An enormous range of functions and libraries provides the necessary calculations or operations in development, the need for designing of own visual elements is avoided [12], [13].

The user application is written in the C\# programming language using the System.Windows.Form libraries that are designed for application development in MS Windows graphical user interface.

\section{Overview of functional requirements}

Workspace:

- The application (hereinafter App.) must work in a clearly defined work environment. This environment must retain information about external links. Specifically, it relates to the device on which the linearization is created and the material for which it is processed. This environment is called "Workspace".

- This environment should also be a common collection for all linearization created on the same machine and material and must somehow archive the results of linearization operations.

- The environment should create linkages between linkages and allow for a general overview.

Linearization:

- App. must be able to display one or more linearization in a common context so that the user can visually compare their values and evaluate errors or deviations. If this requires manipulation of axis, zooming, or zooming data, the application must include these elements.

- App. must under no circumstances modify or otherwise modify the values from loaded linearization. For the results of different operations, it is necessary to create a new linearization. The original (measured) data must always be retained.

Calculations and modification:

- App. must include methods and elements for calculating the deviation $\Delta \mathrm{E}$ by all methods specified in the theory. The user must be able to choose between these methods and, in the case of optional parameters, have the option to enter these parameters.

- App. must be able to transform the $b^{*}$ to $\mathrm{L}^{*}$ axis on any channel to ensure the ability to adapt the application to future knowledge in the art.

File manipulation:

- App. must be equipped with standard file handling controls.

- App. should be able to manipulate disk data without having to wait for the result of this operation (background work).

- App. should periodically back up the work, so that the work can be restored in the event of a fall or a power outage. This operation should not restrict users while working with the application.

- App. should be able to save their work on termination. 


\section{Scenarios and diagrams}

Use case diagrams help determine the scope of the application and present the user interface as the user accesses. It determines the mechanisms and operations that will be required after the application and the individual steps leading to the desired goal. This information is the starting point when designing the application design and ensuring that it fulfils the expected function.
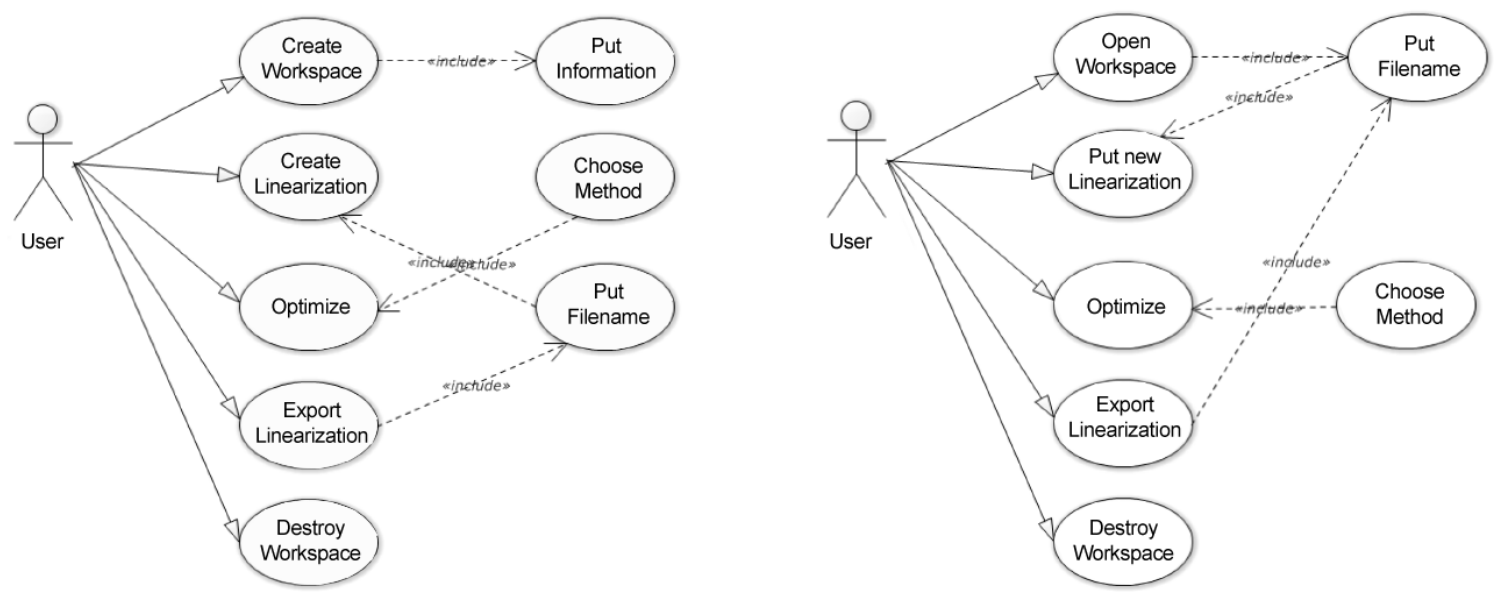

Fig. 2. Use case diagrams: Linearization (left) and Re-Linearization (right).

\subsection{Structure}

The application structure is based on a proven application-programming model for MS Windows. After launch, the main thread creates a custom application window, the System.Windows.Form descendant, which encapsulates all the classes and methods necessary for proper functionality.

\section{Class Workspace}

The instance of the workspace class is generated and extinct depending on the creation or termination of the work environment. It has methods to create custom instances based on user-entered data or data obtained by deserializing the stored instance on disk. It is also able to save to disk, and end its activity if necessary to release the resources for another instance.
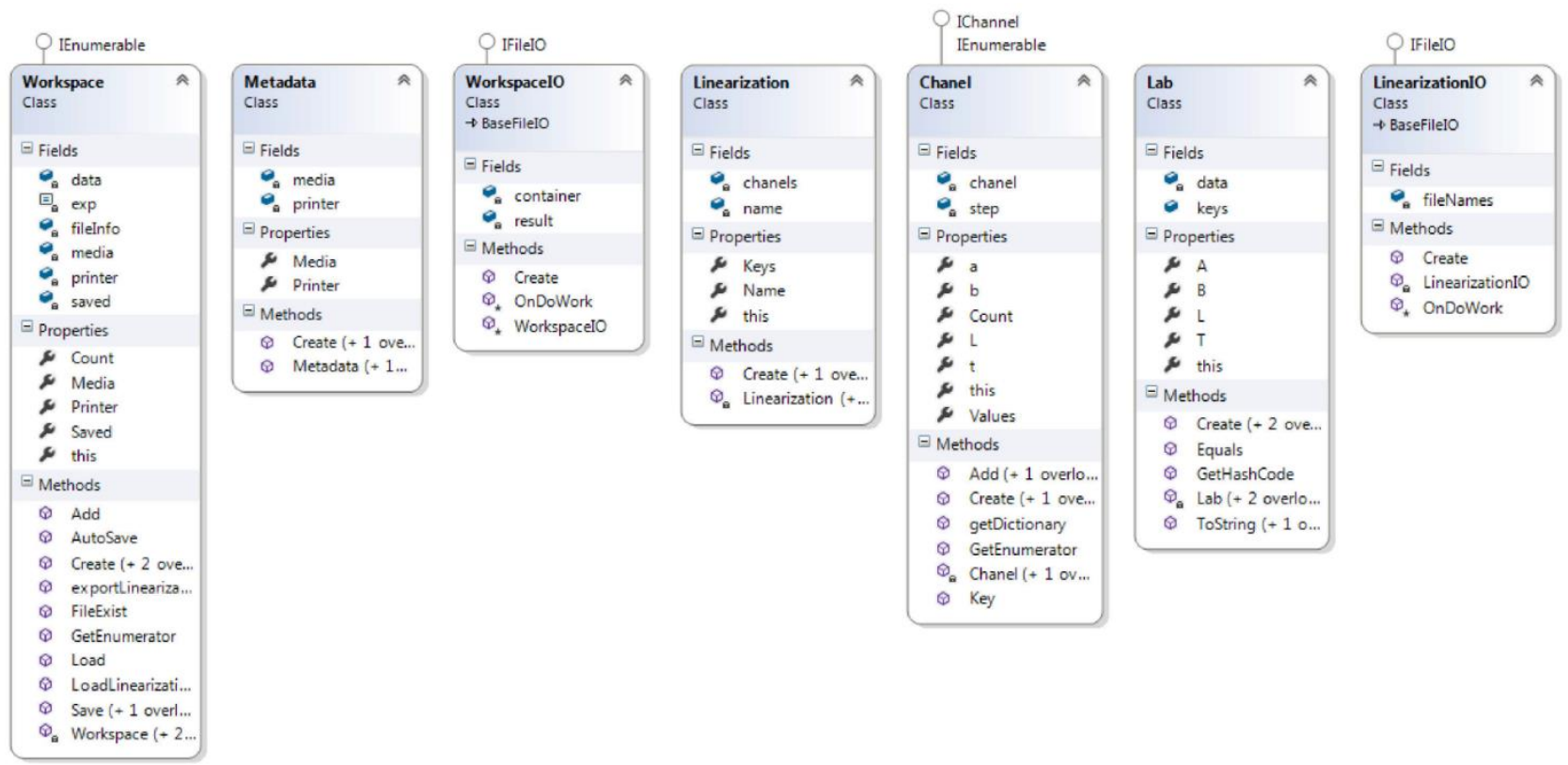

Fig. 3. Design of classes: Workspace, Metadata, WorkspaceIO, Linearization, Channel, Lab, and LinearizationIO.

The Workspace class requires a Metadata class to store printer and material information. It is serialized in parallel with the Workspace class. The Metadata class requires this information and is not able to change it during its existence, just as the workspace is not allowed the Metadata class to change. This enclosure guarantees that data in the work environment is always in relation to one specific machine and material. 
The WorkspaceIO class implements the IFileIO interface, which defines methods for communication with a storage. It contains only the onDoWork method, which allows user to specify data to be written. The rest of the class is inherited by BaseFileIO, which includes methods for manipulating files and working with them. For its function, it needs a container class that encapsulates and structures the data before writing to the repository.

The Linearization class instance serves to preserve just one linearization. This class is the most important element of an application. It divides the linearization on and its data into three parts. The smallest element is the Lab class. This class holds the values of just one measurement, one specific field on a linearization target. The internal vocabulary is used for preservation and there are properties called after axes CIELAB L, A and B (see Fig. 4), these instances are grouped into the Chanel class, which represents one particular measured colour. The Chanel classes are then grouped into one Linearization class.

The LinearizationIO class, as well as its sister class, WorkspaceIO, implements the IFileIO interface and describes how to write to a disk using the OnDoWork method. This class will also inherit BaseFileIO. This procedure provides for the reusability of code for other linearization formats or files that the application might require in its further development.

\section{Interface IDelta}
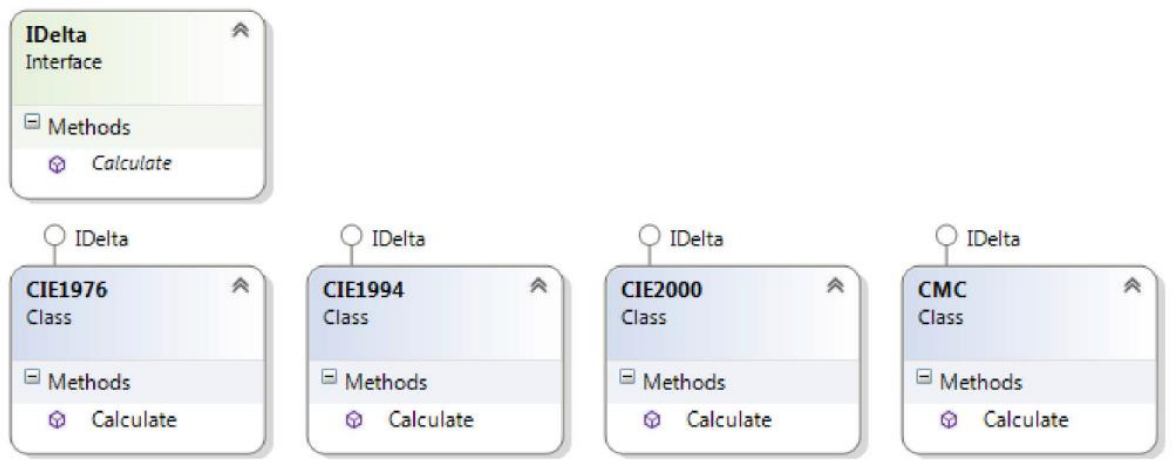

Fig. 4. Interface IDelta including implemented classes.

This interface implements four classes representing the calculation methods $\Delta E$ CIE1976, CIE1994, CIE2000 and CMC. The input parameters are instances of class $L a b$ (at least two) in the form of the list, and the output is a variable of double type that represents the resulting $\Delta \mathrm{E}$.

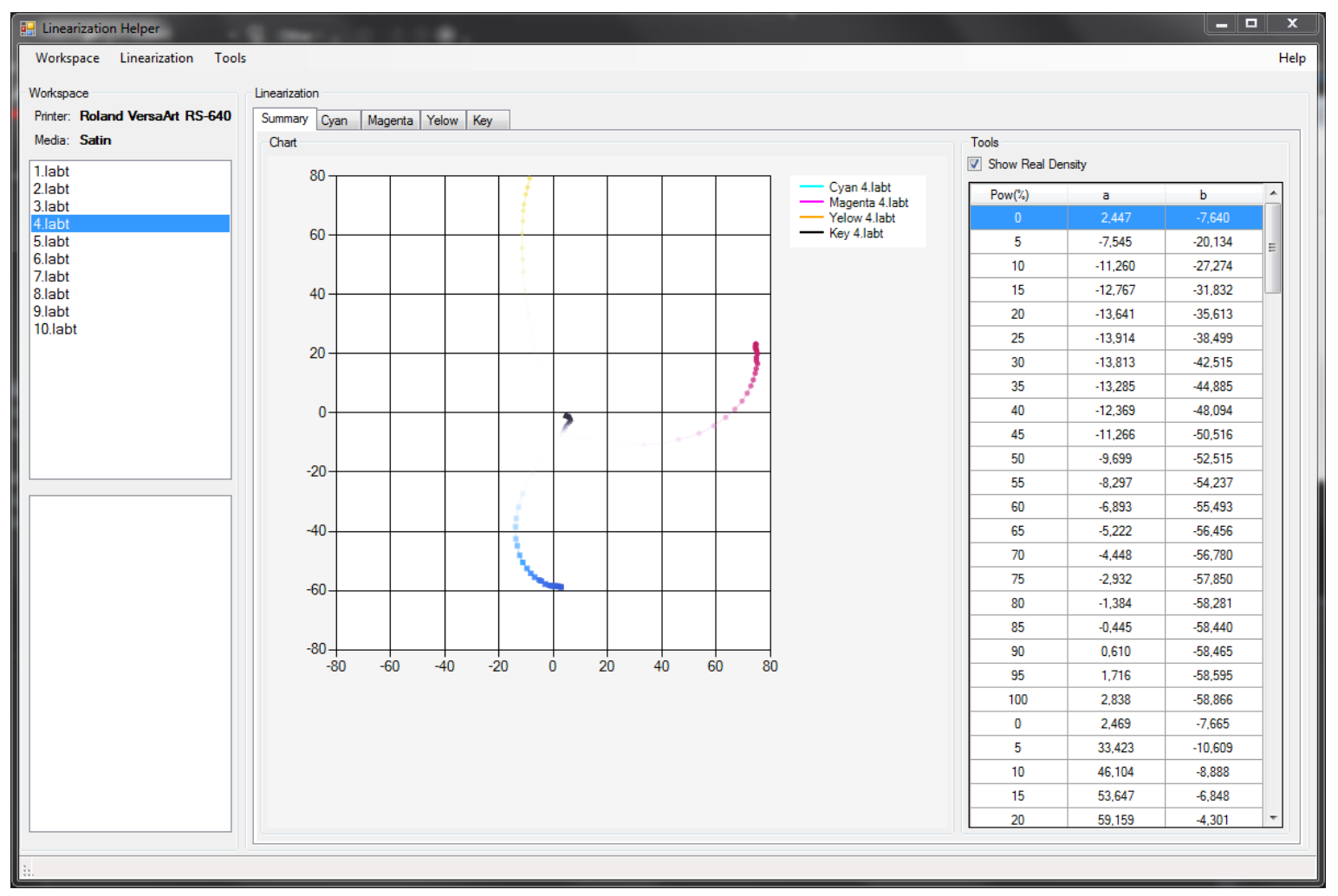

Fig. 5. Design of the main window of the application - Linearization panel - Summary. 
The main application window is divided into the context menu at the top of the window, the status bar at the bottom; see Fig. 5. There are two vertical panels. The Workspace panel works with linearization files and the Linearization panel for visualizing and optimizing data.

Workspace panel contains the name of the printer and the material for which Workspace was created. This data has an informative function and cannot be changed so that Workspace cannot be confused. Two linearization lists are located below and belong to this Workspace. The linearization list at the top contains linearization retrieved from the files and the list at the bottom is created by this application. From these panels user can select individual linearization or groups of linearization, which they will then appear in the right side Linearization panel. It contains several tabs, their number depends on number of colors available for the printer to which linearization is managed. The first tab from left is Summary tab that shows the $\mathrm{C}_{\mathrm{ab}}$ chromatic diagram in which the individual measurements in mutual relationship are placed.

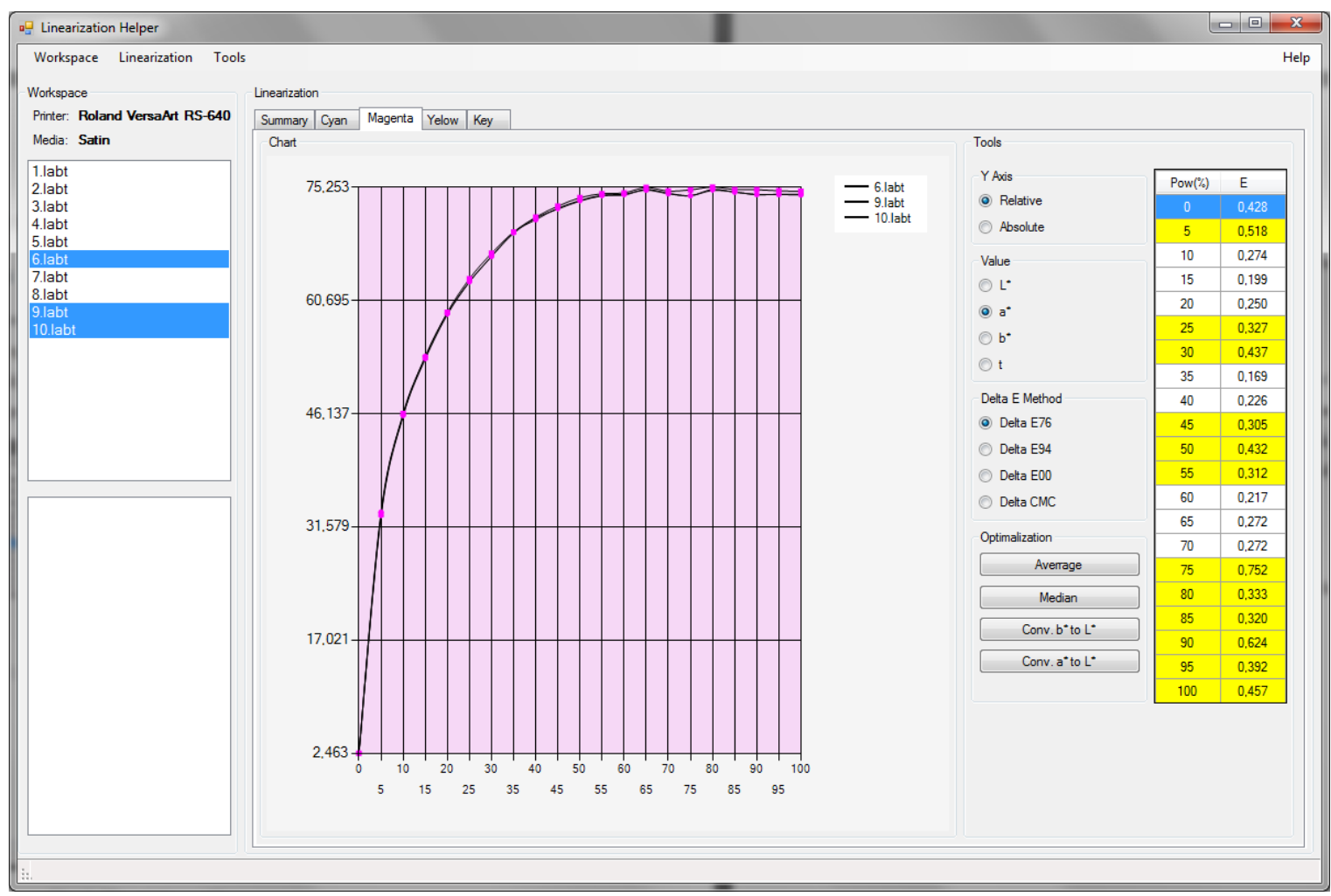

Fig. 6. Design of the main window of the application - Linearization panel - Magenta tab.

The color tabs are labeled with the names of the printer's direct colors for which the linearization was created (see Fig. 6). The tabs represent each color channel for the device. This view allows user to individually process each channel. The color tab content is divided into the Chart view and the Tools section. In the visualization section there is a graph showing one selected CIELAB axis or "Density" in one or more linearization in context. The legend to the right of the graph shows which linearization are currently displayed. On the color tab, there are shown optimization methods that allow correction of linearization or grouping of linearization.

\section{Conclusion}

The aim of this paper was the design of the user application to optimize the calibration of output devices in a digital print environment. It covered collection of spectrophotometric data, their subsequent analysis and design of suitable methods for optimization. The reason for its inception was the serious technological problems in the creation of output ICC profiles just for the needs of digital printing machines and other output devices with CMYK output and with regard to the technology of sublimation of these colors on textile materials.

The analysis of the measured data revealed numerous shortcomings in the resolution of the measuring device, which proved to be insufficient within the parameters set by the manufacturer. Furthermore, it has been found that this device exhibits, on some samples, several times greater variance of the measured data than the frame given by the average and maximum deviation set in the documentation by the manufacturer. Therefore, it is recommended for the above-described printing technologies and especially for sublimation printing to use devices capable of adhering to these tolerances also on textile materials.

During the inspection of the measuring device it was found that the range of data measured for the yellow channel (Y), namely the data for the CIELAB colour space axis L*, is less than the resolution of the measuring device. This feature has helped detect unpredictable results when measuring this colour channel. After further analysis, it was marked as the main cause. In addition, the dependence of error size on the spot colour used and the need to optimize each channel 
individually was determined. The ability to optimize linearization as a whole, i.e. all channels by the same method, proved inappropriate. On the contrary, from the theory and from the results of the statistical analyses, the method of gradual adjustments seems to be the most appropriate.

The main output of this work is, however, an application that we can describe as a librarian editor. This application is able to visualize data both directly in the table, graphs, and has tools to detect some problems that are difficult to detect by default. The application does not aim to compete with professional analytical tools, but only to enable digital printing machines to conduct timely detection of possible errors or undesirable properties of materials or technologies. It was designed so that without any more serious hardware or software entitlement, it could be used in existing operations and able to perform its function. Its main advantage is the ability to work with multiple linearization and to produce averages or medians from the measured values.

It is also capable of transforming axes always to take into account the most significant component of a particular colour channel whose changes will be most noticeable in the event of gradual degradation of equipment or filling or replacement.

The optimization features of the application, which, when called, create new linearization files, allow for easy archiving, the ability to compare each step with a predefined state, and always preserve the original measured values. On the other hand, they do not allow manual intervention in the values to prevent the user from influencing the results and thus the overall re-linearization process.

For future development, it is critical to the application that it has been written with the maximum use of programming methods over the interface. This property gives great potential for its expansion in the future, not only in terms of addition of new procedures and optimization methods, but it is also possible to add new file formats containing linearization and even in different colour spaces without major changes to the code.

\section{References}

[1] Ujiie, H. (2006) Digital Print of Textiles, Woodhead Publishing, ISBN 9781855739512, Cambridge (England).

[2] Dvořáková, Z. (2008) DTP and prepress: complete guide from graphic design to professional printing (in Czech). Computer Press, ISBN 9788025118818

[3] Fernandez-Maloigne, Ch. (2012) Advanced Color Image Processing and Analysis. Springer Science \& Business Media, ISBN 1441961909, NY (USA)

[4] Homann, J (2008) Digital Color Management: Principles And Strategies For The Standardized Print Production, Springer Science \& Business Media, ISBN 9783540693772, Berlin

[5] Schanda, J. (2007) Colorimetry: Understanding the CIE System, John Wiley \& Sons, ISBN 9780470175620, USA

[6] Noyes, Y., Hardeberg, J. \& Moskalev, A. (2000) Linearization Curve Generation for CcMmYK Printing. Proceedings of 8th IS\&T/SID Color Imaging Conference 2000: Color Science and Engineering: Systems, Technologies, Applications, Scottsdale, Arizona (USA), ISSN 2169-2629, ISBN 9781622766284, pp. 247-251, Society for Imaging Science and Technology, Springfield (USA)

[7] http://support.rolanddga.com/docs/documents/departments/technical\%20services/manuals\%20and\%20guides/supp ort_doc_color\%20calibrating\%20a\%20print\%20mode.pdf, (2009). Pozorski, J., Roland DGA Corporation, Color Calibrating a Print Mode: Instructions on How to Linearize Your Printer, Accessed on: 2017-08-24

[8] https://www.xritephoto.com/documents/literature/en/L11-029_color_guide_EN.pdf, (2004). X-rite, The Color Guide and Glossary, Accessed on: 2017-08-24

[9] https://www.yumpu.com/en/document/view/30992971/kind-of-document-quentin, (2006), Kornetka, S., efi GmbH, Re-Linearization vs. L*a*b* Correction: Three Ways to Achieve Good Printer Adjustment, Accessed on: 2017-0824

[10] http://docs.printfactory.cloud/, (2017), PrintFactory Knowledge Base, Accessed on: 2017-08-24

[11]https://my.xrite.com/documents/apps/public/Manuals/EO2UV-QSG_i1Pro_UVcut_User_Manual.pdf, (2016), Xrite, i1Pro2 User Manual, Accessed on: 2017-08-24

[12] https://msdn.microsoft.com/en-us/library/w0x726c2(v=vs.110).aspx, (2017), Microsoft, .NET Framework 4.7, 4.6, and 4.5. Microsoft Developer Network, Accessed on: 2017-08-24

[13]Djambic, G., Kucak, D. \& Fulanovic, B. (2012) Empirical Comparison of Different View state approaches on Performance of ASP.Net Web Applications, Annals of DAAAM for 2012 \& Proceedings of the 23rd International DAAAM Symposium, ISBN 978-3-901509-91-9, ISSN 2304-1382, pp 0733 - 0736, Editor B[ranko] Katalinic, Published by DAAAM International, Vienna, Austria 